\title{
Research on Electronic Controlled EGR System of Diesel Engine
}

\author{
Xinmei Chen \\ School of Power Engineering, Wuhan Institute of Shipbuilding Technology, Wuhan, 430050, China
}

Keywords: Diesels engine, Electronically control system for exhaust gas recirculation, Research.

\begin{abstract}
EGR system is electronically control system for exhaust gas recirculation. This article conducts a detailed research on the EGR control stagy by a systematical elaborate on development procedure, a profoundly discussion to the components and structural software design, an overall consideration to the anti-dereference ability and a thorough analysis for the bench test result of the engines with this system.
\end{abstract}

\section{Introduction}

The main technology in controlling $N O_{x}$ of diesel engine is exhaust gas recirculation, also known as EGR. The main faction of EGR is in the air in tank system, by recycling some exhaust gas back to air in tank system, while jesting and controlling the flow quantity of the exhaust gas. Currently the EGR system has been widely used at home and abroad, and achieved satisfactory results in emission reduction. At present many countries widely used EGR system has to reduce $N O_{x}$ emission effectively, mostly in the indirect and direct deadly light engine.

\section{EGR introduction}

At present stage, the EGR technique is the most common yet effective way to reduce emission. In many countries the EGR is already used in diesel engines of coaches and light trucks, and availed for the heavy trucks. While the basis for EGR is inside exhaust manifold, through the EGR, some burned exhaust gas will be introduced into intake pipe, and mixing with fresh air, then reenter the combustor for combustion. ${ }^{[1]}$ Yet, the cooled EGR exhausted gas can enhance the EGR effects. Which means gas cooler should be used to cool down the exhausted gas, then introduce it into the in tank pipe. Moreover, the mechanism for the EGR is reduce the oxygen concentration in the air mixture and absorb the heat generated through burning, to ensure slow state of burring speed and reduce the heat that generated and finally cut the $N O_{x}$ simian effectively and relive the air pollution issues. But excessive recirculation will increase the first risk of the air mixture or dramatically affect the performance of engines, especially for low-speed running, idle and other similar operations. Therefore during above mentioned situation, electronic control unite will issue timely control commands and cut off ERG valve as quick as possible to prevent exhaust gas from pretending to the recirculation process. But the electronic control unite have to issue timely commands to open EGR valve and ensure some exhaust gas will re-attend the reciprocation progress if the engine is over the engine speed limitation or engine loading, and the temperature has already reached the specification. And the amount of exhaust gas attending the recirculation is decided by specific load, speed or temperature of the cooling liquid, but enough to assure the attempt of reducing the $N O_{x}$ estimate in exhaust gas. $^{[2]}$

\section{Components of the electronic controlled EGR system for diesel engine}

The main components of electronic controlled EGR system are stepped motor, EGR velvet, sensor and ECU. the main purpose for the ECU is to ensure the data collection ,conduct proper command company to the operation situation, chive coordinate between each sub program and take appropriate control of the output signal .contents of sensor are the singles for engine temperature, rotational speed, machine temperature and pressure and temperature of the machine, and accelerator 
pedal positioned. the rotary motion of spate motor is transformed into linear motion by screw mechanism, then it will push the ERG valve and reset spring to enable balancing to assure stability of the EGR valerian specified position According to the experiments, maps of EUG positions will be stored in $\mathrm{ECU}^{[3]}$. So during the actual running of the engine, ECG can corrected the sing on responding to the actual running information and basic single like loading and temperatures, and figure out the correspondence EGR position for current operation condition, then convert it into number of controlling pulses for the step motor. The expanded controlling pulses power can achieve the running of step motor and accurate control the position of ECU valve. While, feedbacks from the sensors in the specific locations will conduct reasonable final correction to the controlled figures.

\section{The hardware architecture of the control unite}

The hardware architecture of the control unite is as shown in Fig 1.

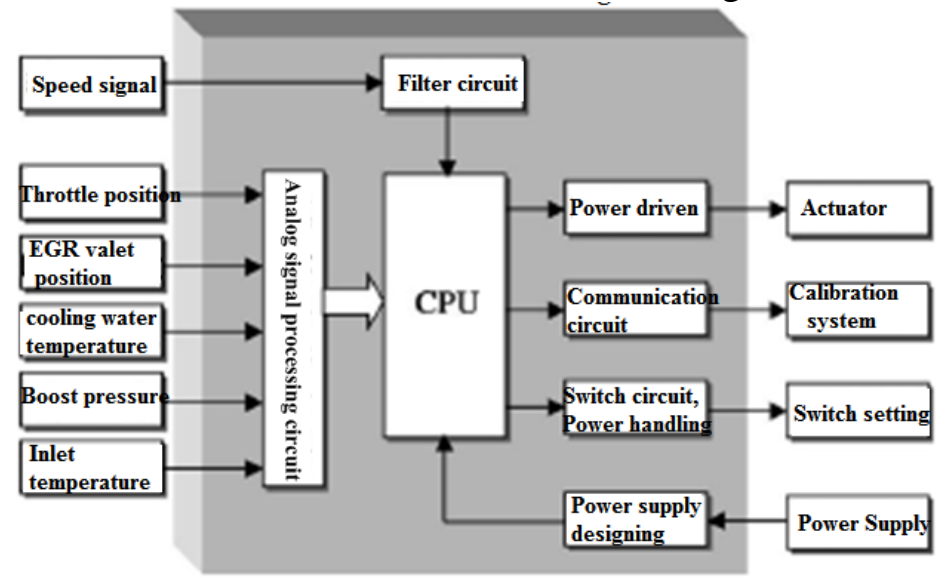

Fig. 1

The main choice for CPU in the system is ARM2119 microcontroller, is to achieve the stepper motor actuator drive by timely acquisition of inlet pressure, water and soil temperature, analog signal and speed signal, and regard the output signal as PWM signal. Meanwhile, under the function of serial bus, the communication between control and monitoring system can be realized. Once the analog signal is filtered, enlarged and limited, it can achieve A/D conversion. The specific processing circuit can be represented by Fig 2.

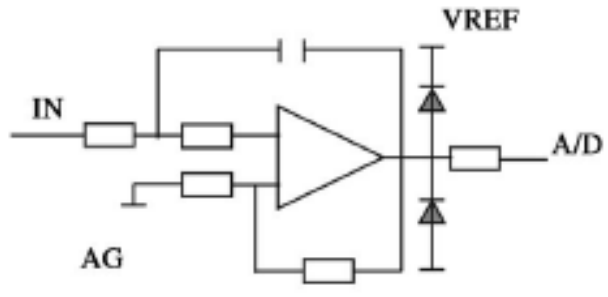

Fig. 2

The sine waves that outputted by in electromagnetic speed sensor, is known as speed. The relationship between the actual output frequencies, voltage signal, engine speed signal, are positive proportion. Meanwhile, it will be sent to the CPU after filtering, amplifying and shaping, which is an important measure factors for engine speed. The main used in the implementation in this system the stepper motor, the multiple index like speed, load and hydrology and other environmental parameters can ensure ECU has higher accurately determine rate of ECU than EGR for this time, And output signal can corresponding control of to steering and step number of the stepper motor, the stepper motor can achieve the changes form rotary motion to linear motion with the assistance of connections, and push the EGR valve by stem reset spring compression, and then conduct reasonable control of opening, which means control the amount of exhaust gas to reticulate into the system. 
The stepping motor is the important loop control unit during the process of changing the electric pulse signal into angular displacement or line displacement. In a state of non overload, the actual speed of the motor and the position of the final stop are only related to the pulse signal frequency and pulse number, and will not be affected by changes of loading. Simply speaking, the increase in the pulse signal will result corresponding rotate angle cloth of the motor. The step motor has been widely used in many kinds of control fields such as speed or position for this kind of linear relation, and the other characteristic such as periodic errors. Among them, the ring pulse, signal distribution and power amplification, load and so on are the important components of the stepper motor drive control system.

1). Pulse Signal

CPU will produce pulse signals, in general situation, duty cycle of pulse signal is controlled between [4] 0.3-0.4. The duty cycle will become larger, if the actual speed of the motor is relatively higher.

\section{2). Power amplifier}

The drive system is an important part of the power amplifier. Under certain rotational speed, the torque of the stepping motor directly related to its dynamic average current. The motor torque will gradually increase as the average current increased ,so in order to increase the average current, the drive system need to overcome the EMF as much as possible. Different corresponding drive models have to chosen for different occasions.

\section{The software design for electronic control EGR system.}

16 microcontroller is taken as the core of controller for diesel engine electronic control EGR system as the, while, the control software need to be programmed by assembly language. Among them, the main control program is to achieve following targets such as measurement and determine the engine conditions, handling and identification to the processing programs etc, after power initialed. The main function of the controlling program is to judge engine' $s$ various working conditions which are done mostly according to the position and speed of the throttle. Since the main control program is a cycle process, in the actual operation of the engine, the main control program always is a cycling executing state. It can complete predetermined action in different conditions through the following stages; determine the actual working situation of the engine through corresponding to A / D conversion subroutine , then use the step motor drive and electromagnetic valve control subroutine to output the corresponding control command.

\section{Analysis on control method of electronic controlled EGR system}

In the actual operation of the engine, the waste recycling rate will affect the performance of its work, it will be difficult to reduce $N O_{x}$ emissions if the amount of exhaust gas is relatively small. However, if the discharge is too large, then it will lead to an unstable state, deterioration of the performance of the engine itself, ${ }^{[5]}$ If the EGR rate increased, the $N O_{x}$ will be effectively reduced, but some other index like fuel consumption, and etc will raise accordingly. Generally speaking, engine speed and load are can determine the rate of EGR, so in a variety of conditions suitable EGR rate should be fully measured and consider the power and economy, and even emissions.

$i$. When the engine is low in state and load, then it will be very unstable phenomenon since the fuel injection quantity and combustion is not high at the same time. At this point, the exhaust gas recirculation rate needs to be reduced.

But when the engine is high in state and load, in order to achieve high output power, the exhaust gas recirculation rate needs to be reduced.

ii .If the engine is idle, and the combustion temperature is relatively low, and the $N O_{x}$ emissions is relatively small, too ensure the steady operation of the engine, the exhaust gas recirculation should be cut off. 
iii.If the temperature of engine's cooling water is not high, then the mixture of oil and gas will be difficult to spread evenly in the cylinder, which will make the combustion to unstable state, so the exhaust gas recirculation should be cut off. However, if the temperature of the cooling water is increasing, the amount of exhaust gas recirculation should be increased accordingly.

iv. When starting the generator, to ensure the stability of the generator and smooth starter, the exhaust gas recirculation should be cut off

$\mathrm{v}$.When the temperature of intake air is not high, which will also reduce the temperature of cylinder internal combustion? In this situation, the proper reduction of exhaust gas recirculation is needed to ensure that the actual combustion process can be run properly.

vi.When temperature of the engine's cooling water lower than 35 degrees Celsius or higher than 90 degrees Celsius. In order to prevent the instability of combustion, the EGR system should be cut off in a timely manner.

\section{Anti jamming mode of electronic control EGR system for diesel engine}

1). Since the power to the diesel engine is mainly provided by the battery, when charging, fluctuations in the power are the main disturbance. In order to avoid this kind of interference effectively, the system uses a high performance power supply voltage regulator, and the multilevel filter is carried out at the same time.

2). Since the system drive actuator is required for high voltage and large current, and the generation of instantaneous current will also be introduced into the control system through the power drive circuit, then it may produce certain disturbance. Therefore, in the design work, photoelectric coupling - power amplifier circuit is used to ensure effective control of the signal and the driving signal in electrical in a certain extent and enhance the ability to resist interference.

3). Since the EGR system, analog signals in the form of cable to introduce the control system, transmission will be certain interference. Therefore, in the design of the lines, simulate the circuit between the digital and the separations of the single points are needed only the circuit board will be connected. Meanwhile, steeply leading edge of the digital signal, so once in a state of high frequency, line class the distributed capacitance and leakage coupling will be needed, will produce signal interference at the end of the analog. Therefore, in the design process, a relatively far distance is needed between digital signal and analog signal to avoid the emergence of large interference.

\section{Research and analysis of concrete test}

In this paper, proposed an electronic control system by own invention, and used in the test of the engine bench. The final result is relatively ideal. Because the electronic control system can give full play to its own electronic control function, and satisfied all the requirements during the engine operating in various conditions. In addition, despite the short actual running time, this electronic control system can response rapidly that also laid a solid foundation for the safety of the engine operation. Meanwhile this system is applied to four cylinder turbocharged diesel engine with direct injection in the cold light, and conducted analysis on the emission performance and economic performance result. The final conclusion is this system can conduct suitable adjustment to the EGR rate and control the EGR valve according to the specific control strategy.

\section{Conclusion}

In summary, with the popularization and application of the diesel engine, the $N O_{x}$ emission of the waste gas is gradually increasing, which further aggravate the degree of air pollution. In order to effectively alleviate the above problems, the electronic control EGR system can be used in the diesel engine, which will effectively reduce the $N O_{x}$ emissions of waste gas, and alleviate the air pollution issues. And the application of the system in the practice of the effect is also very good, so, it is worth promoting. 


\section{References}

[1] Su Dong ,GongYuanming,Li Chaoyang alt,etc Design and application of electronic controlled EGR bench test, Journal of Shanghai University of Engineering Science, 2013,27(3):202-207.

[2] Zhan Xiongwei, Chen Wenchao FeiTeng, alt, etc. Research on control and matching of EGR diesel engine in China, Modern vehicle power, 2015(4):10-13.

[3] Long Hsisheng, Pneg Yuming, LiDayong alt, etc. Simulation analysis based on the difference of EGR rate difference of each cylinder of diesel engine, Mechanical engineering and automation, 2015(1):52-53.

[4] Zhang Ke, Chen Xiying, ZhangQingalt, etc. Experimental study on the performance and emission of 485 diesel engine based on EGR injection parameters, Proceedings of the 2013 annual conference of the China Society for combustion and energy conservation of China internal combustion engine, 2013:1-6.

[5] Wang Kejie Zhou Wenhua,Nie Fei alt, etc. Research on fault diagnosis of EGR system, Mechanical and electrical engineering, 2016,33(2):212-216. 